\title{
Molecular characterization of hard ticks from Romania by sequences of the internal transcribed spacers of ribosomal DNA
}

\author{
L. Chitimia • R. Q. Lin • I. Cosoroaba • P. Braila • \\ H. Q. Song • X. Q. Zhu
}

Published online: 13 August 2009

(C) Springer-Verlag 2009

\section{Erratum to: Parasitol Res DOI 10.1007/s00436-009-1474-1}

The original version of this article unfortunately contained a mistake. The title was incorrect. The title should be "Molecular characterization of hard ticks from Romania by sequences of the internal transcribed spacers of ribosomal DNA". Also, Dermanyssus gallinae was mistaken as soft tick in several places. The correct version of the article is shown below.

Abstract In the present study, four hard tick species and one mite species, namely, Dermacentor marginatus,

The online version of the original article can be found at http://dx.doi. org/10.1007/s00436-009-1474-1

L. Chitimia $\cdot$ R. Q. Lin $\cdot$ H. Q. Song $\cdot$ X. Q. Zhu $(\bowtie)$

College of Veterinary Medicine,

South China Agricultural University,

483 Wushan Street, Tianhe District,

Guangzhou, Guangdong Province 510642,

People's Republic of China

e-mail: xingquanzh@scau.edu.cn

L. Chitimia

e-mail: lidychitimia@yahoo.com

L. Chitimia

Institute for Diagnosis and Animal Health,

Bucharest, 63 Dr. Staicovici, sector 5,

050557 Bucharest, Romania

I. Cosoroaba

Department of Parasitology, Faculty of Veterinary Medicine,

Calea Aradului 119,

300645 Timisoara, Romania

P. Braila

Sanitary Veterinary Direction, Food Security Resita,

Resita, Romania
Haemaphysalis punctata, Haemaphysalis parva, Ixodes ricinus, and Dermanyssus gallinae, from south-western Romania were characterized genetically by the first (ITS-1) and second (ITS-2) internal transcribed spacers (ITS) of nuclear ribosomal DNA (rDNA), using a hard tick, Haemaphysalis longicornis, from China for comparative purposes. The ITS rDNA was amplified by polymerase chain reaction (PCR) and sequenced from individual ticks. The lengths of the ITS-1 sequences were 238-1819 bp, and the lengths of ITS-2 were 137-1695 bp, respectively, for all ticks sequenced. While sequence variation within a hard tick species was $0-1.5 \%$, nucleotide differences between hard tick species ranged $2-25.2 \%$, indicating that ITS rDNA sequences provide genetic markers for the differentiation of hard ticks from Romania. Hence, a PCR-linked restriction fragment length polymorphism approach was developed for their unequivocal differentiation based on ITS-1 rDNA. This is the first characterization of ticks from Romania using a genetic approach, which provides the foundation for further studies on ticks in Romania and has implications for studying the population genetic structure of the Romanian ticks and for identification and differentiation of closely related ticks.

\section{Introduction}

Ticks are obligate hematophagous ectoparasites of a wide variety of terrestrial vertebrates, including amphibians, reptiles, birds, and mammals. Ticks form a suborder (Ixodida) in the order Parasitiformes of the subclass Acari. This suborder consists of two families, the Argasidae (soft ticks) and the Ixodidae (hard ticks). The Ixodidae is split into five subfamilies (Ixodinae, Amblyomminae, Haemaphysalinae, Hyalomminae, and Rhipicephalinae). An important division of the Ixodidae consists of prostriate (Ixodinae) and 
metastriate ticks (the other four subfamilies; Black and Roehrdanz 1998). Ticks are of considerable economic importance throughout Romania due to both the direct effects of feeding on livestock and for transmitting tick-borne diseases (e.g., Babesia, Rickettia, tick-borne encephalitis).

The specific identification of ticks at any developmental stages by morphology is not always possible due to the bad preservation of the specimens that can preclude correct identification by using morphological characters. Furthermore, detailed morphological descriptions of the immature stages remain unknown for most tick species. To circumvent somehow these kinds of difficulties, several approaches using different genetic makers have been evaluated for the identification and phylogenetic studies of ticks (Wesson et al. 1993; McLain et al. 1995; Zahler and Gothe 1997; Poucher et al. 1999; Fukunaga et al. 2000; Hlinka et al. 2002; Shaw et al. 2002; Rees et al. 2003; de Rojas et al. 2007; Marrelli et al. 2007). Concerning the public health aspects, molecular methods applied to the diagnosis of ticks can be useful and practical. DNA extracted from ticks harvested at focus of tickborne disease sites could be used for identification of both the agent and the vector species (Marrelli et al. 2007).

The first (ITS-1) and second (ITS-2) internal transcribed spacers (ITS) of ribosomal DNA (rDNA) are useful genetic markers for defining species boundaries and for inferring phylogenies because there tends to be little intraspecific variation but considerable interspecific variation (Barker 1998; Zhu et al. 1998, 2007). Highly conserved rDNAs flank both ITS regions so it is relatively simple to amplify them by polymerase chain reaction (PCR; Murrell et al. 2001). ITS-2 rDNA has been sequenced extensively in ticks to study closely related species (McLain et al. 1995; Zahler et al. 1995; Zahler and Gothe 1997; Barker 1998; Fukunaga et al. 2000; Murrell et al. 2001) and other parasites (Li et al. 2005; Zhu et al. 1998; 2007). Barker (1998) is the most pertinent of these to the present study because it dealt with intraspecific variation in the ITS-2 of Rhipicephalus and Boophilus spp. However, prior to present study, there had been no studies of ticks in Romania using well-defined DNA markers. Therefore, by using ITS rDNA as genetic marker, the objective of the present study was to characterize samples representing four hard tick species and one mite species, namely, Dermacentor marginatus, Haemaphysalis punctata, Haemaphysalis parva, Ixodes ricinus, and Dermanyssus gallinae, from south-western areas of Romania, and using a hard tick, Haemaphysalis longicornis, from China for comparative purposes.

\section{Materials and methods}

\section{Parasites}

Ticks were collected from naturally infested sheep from south-west areas of Romania. After collecting, the ticks were transported to the Laboratory of Department of Parasitology, University of Agriculture and Veterinary Medicine from Timisoara, Romania, and processed. Ticks were identified morphologically and then conserved in absolute ethanol. The tick species, with their codes and geographical origins and GenBank ${ }^{\mathrm{TM}}$ accession numbers for their ITS- 1 and ITS- 2 sequences, are listed in Table 1.

\section{Extraction and purification of genomic DNA}

Genomic DNA (gDNA) was extracted and purified from the complete body of adult ticks. Prior to DNA extraction, each sample was washed in decreasing concentrations of alcohol $(70 \%, 50 \%, 30 \%, 10 \%) 1 \mathrm{~h}$ for each concentration and finally in double distilled water for $1 \mathrm{~h}$. Genomic DNA was extracted from individual ticks according to the

Table 1 Tick and mite species used in the present study with their codes, geographical origins in Romania, and GenBank ${ }^{\mathrm{TM}}$ accession numbers for their ITS-1 and ITS-2 sequences

\begin{tabular}{lllll}
\hline Species & Code & Geographic origin & Accession no. ITS-1 & Accession no. ITS-2 \\
\hline Ixodes ricinus & IRP1, IRP2 & Pişchia & FN296266 & FN296277 \\
Dermacentor marginatus & DMC & Carasova & FN296263 & FN296273 \\
Dermacentor marginatus & DMMN & Moșniţa Nouă & FN296267 & FN296278 \\
Dermacentor marginatus & DMST & Sacoșu Turcesc & FN296259 & FN296269 \\
Dermacentor marginatus & DMCV & Covaci & FN296265 & FN296275 \\
Haemaphysalis punctata & HPST & Sacoşu Turcesc & FN296264 & FN296274 \\
Haemaphysalis punctata & HPMN & Moșnița Nouă & FN296262 & FN296272 \\
Haemaphysalis parva & HPAP1, HPAP2 & Pișchia & FN296280 & FN296283 \\
Haemaphysalis longicornis & HL1, HL2 & Gansu, China & FN296261 & FN296271 \\
Dermanyssus gallinae & DGC & Carasova & FN296258 & FN296258 \\
\hline
\end{tabular}




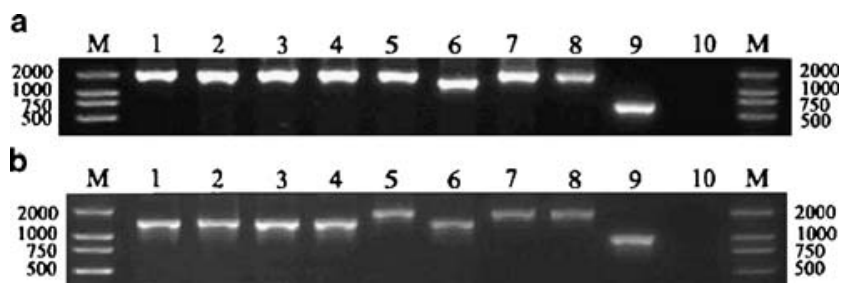

Fig. 1 Agarose gel electrophoresis of ITS-1 (a) and ITS-2 (b) amplicons of tick samples from different places in south-western areas of Romania. Lanes 1-9 represent PCR products amplified from $D$. marginatus (lanes 1-4), H. longicornis (lane 5), H. parva (lane 6), $H$. punctata (lanes 7, 8), and I. ricinus (lane 9) samples. Lane 10 represents negative control. $M$ represents DL2000 DNA size marker

procedure described by Zahler et al. (1995) with some modifications made by Marrelli et al. (2007). Individual ticks were frozen and ground to a fine powder under liquid nitrogen in a mortar. The powder was resuspended in $400 \mu 1$ of TET buffer $(50 \mathrm{mM}$ Tris-HCl, pH7.5, $25 \mathrm{mM}$ EDTA, $100 \mathrm{mM} \mathrm{NaCl}$, and $0.4 \%$ Triton X-100) and $30 \mu \mathrm{l}(20 \mathrm{mg} / \mathrm{ml})$ of proteinase $\mathrm{K}$. After incubation at $56^{\circ} \mathrm{C}$ overnight, the DNA was extracted by shaking in phenol/chloroform/isoamylic alcohol (25:24:1) and precipitated by adding 2 volumes of icecold ethanol for 4-6 h. After centrifugation $(12,000 \times g$ for $5 \mathrm{~min})$, the pellet was resuspended in $50 \mu \mathrm{l}$ of TE $(10 \mathrm{mM}$ Tris, pH8.0, and $1 \mathrm{mM}$ EDTA), and the DNA was used as template for PCR.

Enzymatic amplification of the ITS-1 and ITS-2 rDNA

The ITS-1 rDNA plus primer flanking $18 \mathrm{~S}$ and $5.8 \mathrm{~S}$ rDNA was amplified by PCR from hard tick gDNA using primers TITS1F (5'-TCATAAGCTCGCGTTGATT-3') and TITS1R (5'AGCTGGCTGCGTTCTTCAT-3'), whereas the ITS-2 rDNA, including primer flanking $5.8 \mathrm{~S}$ and $28 \mathrm{~S}$ rDNA sequences, were amplified using primers TITS2F1 (5'-CGAGACTTGGTGT GAATTGCA-3') and TITS2R1 (5'-TCCCATACACCA CATTTCCCG-3'). Primers ITSDGF (5'-AGAGGAAGT AAAAGTCGTAACAAG-3') and ITSDGR (5'-ATATGCT TAAATTCAGGGGG-3') were used to amplify entire ITS from the poultry red mite $D$. gallinae. Polymerase chain reactions were performed in $25 \mu \mathrm{l}$ volumes, including $0.5 \mu \mathrm{l}$ of each $50 \mu \mathrm{M}$ PCR primer, $0.5 \mathrm{U}$ of rTaq DNA polymerase (Takara), and with $\mathrm{MgCl}_{2}$ concentration of $2 \mathrm{mM}$ under the following conditions: $95^{\circ} \mathrm{C}$ for $5 \mathrm{~min}$ (initial denaturation); followed by 40 cycles of $95^{\circ} \mathrm{C}, 45 \mathrm{~s}$ (denaturation); $55^{\circ} \mathrm{C}$, $1 \mathrm{~min}$ (annealing); $72^{\circ} \mathrm{C}, 90 \mathrm{~s}$ (extension); and a final extension of $72^{\circ} \mathrm{C}$ for $5 \mathrm{~min}$. Negative controls (no template) were always run simultaneously with PCR experiments. Successful amplification was determined by electrophoresing $2 \mu 1$ of the PCR amplicons on $1 \%(w / v)$ agarose gels $(1 \times$ TBE) and visualizing with ethidium bromide staining. The DL2000 DNA size marker (Takara) was included on the agarose gels to estimate the lengths of amplificons.
Cloning and sequencing of ITS- 1 and ITS-2 rDNA

The purified PCR products were ligated with $\mathrm{pGEM}-\mathrm{T}^{\mathrm{TM}}$ Easy vector (Promega) according to the manufacturer's recommendations, and constructs were then transformed into JM109 competent cells (Promega). The recombinant bacterium was screened, identified by PCR amplification, and then sequenced by BioSun Biotechnology Company using ABI 377 automated DNA sequencer (BigDye Terminator Chemistry) from both directions using the T7 and SP6 sequences flanking the cloning site as primers.

Polymerase chain restriction-linked restriction fragment length polymorphism

Purified ITS-1 PCR products were digested directly with 10 units $(1 \mu \mathrm{l})$ of restriction endonuclease AflI (Promega) in a volume of $20 \mu \mathrm{l}$ at $37^{\circ} \mathrm{C}$ overnight according to the manufacturer's recommendations. Restriction fragments were separated on $1.5 \%$ agarose gels stained with ethidium bromide and photographed using a gel documentation system (UVItec). The sizes of fragments were estimated using the DL2000 DNA size marker (Takara).

\section{Results and discussion}

Genomic DNA was isolated from one to four individuals representing each of the four hard tick species and the poultry red mite from south-west areas of Romania and one species from China (Table 1). The ITS-1 and ITS-2 fragments of D. marginatus, $H$. punctata, H. longicornis, H. parva, and I. ricinus amplified from each sample using primers TITS1F and TITS1R, TITS2F1, and TITS2R1 were between approximately 750 and 2,000 bp in length (Fig. 1). The entire ITS fragment of $D$. gallinae amplified from each sample using primers ITSDGF and ITSDGR was approximately 500 bp in length (Fig. 2), as reported by de Rojas et al. (2007). In no case was product amplified from no-DNA sample. All samples appeared as a single band (Figs. 1 and 2).

The lengths of ITS- 1 were $1,639-1,642$ bp for $D$. marginatus and 1,556-1,819 bp for $H$. punctata, with slight variation in length and nucleotides being detected within a species. For $H$. longicornis, the ITS-1 was

Fig. 2 Agarose gel electrophoresis of ITS PCR product of D. gallinae sample. Lane 1 represents the $D$. gallinae s ample, whereas lane 2 represents negative control. $M$ represents DL2000 DNA size marker

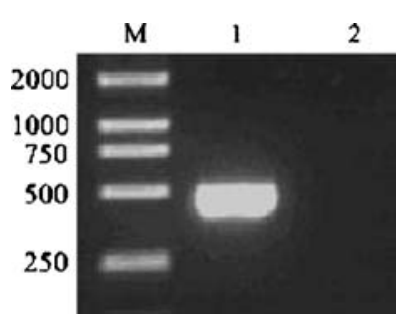


Fig. 3 RFLP analysis of ITS-1 from D. marginatus (lanes 1-4), H. longicornis (lane 5), $H$. parva (lane 6), H. punctata (lanes 7,8 ), and $I$. ricinus (lane 9) samples using restriction enzyme $A f l 1$. $M$ represents DL2000 DNA size marker

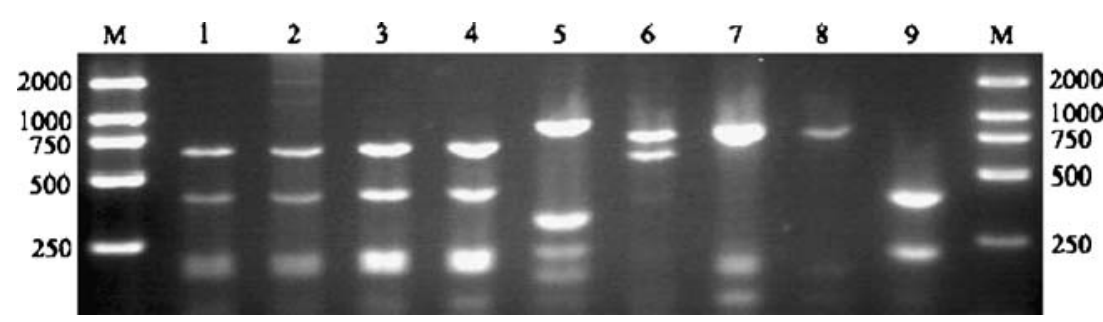

1,572 bp in length. For H. parva, its ITS-1 was 1,542 bp in length, whereas the length of ITS-1 was $343 \mathrm{bp}$ for $I$. ricinus and $238 \mathrm{bp}$ for $D$. gallinae. The $\mathrm{G}+\mathrm{C}$ contents in ITS-1 sequences of all hard ticks were $54.73-60.66 \%$, whereas the poultry red mite $D$. gallinae had $40.76 \% \mathrm{G}+\mathrm{C}$.

ITS-2 rDNA was 1,695 bp in length for D. marginatus and 1,107-1,109 bp for H. punctata, 1,602 bp for H. longicornis, $1,138 \mathrm{bp}$ for $H$. parva, $649 \mathrm{bp}$ for and I. ricinus, whereas the ITS-2 of the poultry red mite $D$. gallinae was extremely short, only $137 \mathrm{bp}$. The $\mathrm{G}+\mathrm{C}$ contents of ITS-2 also varied, being $47.61 \%$ for $I$. ricinus, $55.62 \%$ and $62.92 \%$ for other hard ticks, and $35.04 \%$ for D. gallinae.

Hlinka et al. (2002) studied the secondary structure of ITS-2 from species of the six main lineages of hard ticks (family Ixodidae). The ITS-2 of these ticks varied in length from $679 \mathrm{bp}$ in Ixodes scapularis to 1,547 bp in Aponomma concolor. Nucleotide contents varied also, and the ITS-2 of ticks from the Prostriata lineage had $46-49 \% \mathrm{G}+\mathrm{C}$, whereas ITS-2 sequences of ticks from the Metastriata lineage had $61-62 \% \mathrm{G}+\mathrm{C}$. Marrelli et al. (2007) determined the ITS-2 sequences of 15 hard tick species of the genus Amblyomma found in Brazil. Lengths of these ITS-2 sequences ranged from 956 to $1,207 \mathrm{bp}$, whereas $\mathrm{G}+\mathrm{C}$ contents varied from $62.4 \%$ to $66.9 \%$.

The two samples representing $I$. ricinus from the same location (Pişchia) had identical ITS-1 and ITS-2 sequences. Similarly, the two samples representing $H$. parva from Pișchia also had identical ITS sequences. No nucleotide variation was detected between the ITS sequences of the two samples representing $H$. longicornis from Gansu, China. Sequence variation was $0.7-1.4 \%$ in ITS- 1 rDNAs representing $D$. marginatus ticks from four different geographical locations. For $H$. punctata, sequence variation in ITS-1 was $1.5 \%$ between ticks from two different places. For D. marginatus, sequence variation in ITS-2 rDNA was $0.9-1.2 \%$ among ticks from four different geographical locations. For $H$. punctata, variation in the ITS-2 sequence was $0.4 \%$ between ticks from two different places, whereas sequence differences among different species ranged from $3.8 \%$ to $21.9 \%$ for ITS-1 and from $2.0 \%$ to $25.2 \%$ for ITS-2, significantly higher than nucleotide variation within a species, indicating that both ITS-1 and ITS-2 rDNA sequences provide specific markers for the accurate identification and differentiation of ticks.

Restriction maps were constructed for the ITS-1 sequences of hard ticks examined in the present study, and several sites existed for a number of common restriction endonucleases (not shown). Comparison of undigested ITS-1 PCR products revealed that I. ricinus could be distinguished from the other hard tick species on agarose gels based on small-sized product ( $\sim 750 \mathrm{bp})$, but D. marginatus could not be distinguished from $H$. punctata based on product size (both $\sim 2,000$ bp; Fig. 1). As expected from the restriction maps for ITS-1 rDNA (not shown), PCR-linked restriction fragment length polymorphism (PCR-RFLP) analysis of PCR products with $A f l \mathrm{I}$ allowed the differentiation of the five hard tick species because they had different restriction banding patterns (Fig. 3).

The ITS-2 of hard ticks has apparently evolved mostly by increases and decreases in length of the nucleotide sequences, which caused increases and decreases in the length of stems of the secondary structure. This is most obvious when stems of the secondary structures of the Prostriata (Ixodes spp.) are compared to those of the Metastriata (all other hard ticks). Increases in the size of the ITS-2 may have been caused by replication slippage which generated large repeats, like those seen in Haemaphysalis humerosa and species from the Rhipicepalinae lineage and the small repeats found in species from the other lineages of ticks (Hlinka et al. 2002).

In conclusion, the present study represents the first genetic characterization of ticks from Romania using a molecular approach, and the established PCR-RFLP assay would provide a useful molecular tool for the differentiation of closely related hard ticks in Romania, which has implications for studying the population genetic structure of the Romanian ticks and for identification and differentiation of closely related ticks.

Acknowledgments This work is supported by a grant from the Program for Changiiang Scholars and Innovative Research Team in University (Grant No. IRT0723) to XQZ. LC was supported by a postdoctorial fellowship from Guangdong Provincial Government, China. The experiments comply with the current laws of the countries in which the experiments were performed. 